\title{
Studententag zum Deutschen Zahnärztetag: Wie man ein Tiger- gulasch kocht - zahnärztliche Kommunikation einmal anders
}

\author{
Tigergulasch zuzubereiten ist gar nicht so sehr eine Frage des Garens und Würzens, sondern hängt eher \\ davon ab, den Tiger erst einmal zu fangen... Der Studententag zum Deutschen Zahnärztetag 2017 (Sams- \\ tag, 11. November, ab 10 Uhr, Raum Spektrum) widmet sich in diesem Jahr einem auf den ersten Blick \\ ungewöhnlichen Thema.
}

$\mathrm{O}$ ganisiert wird der Studententag wieder von der Deutschen Gesellschaft für Zahn-, Mund- und Kieferheilkunde (DGZMK), dem Bundesverband der Zahnmedizinstudenten in Deutschland (BdZM) und der Initiative young dentitsts $\left(\mathrm{yd}^{2}\right)$. „Der Studententag soll in diesem Jahr neben informativen Veranstaltungen auch einen fortbildenden Teil beinhalten. Den fortbildenden Teil möchten wir vor allem mit der Veranstaltung am Vormittag,Zahnärztliche Kommunikation - wie man ein Tiergulasch kocht...' abbilden.“, so Lotta Westphal vom Vorstand des BdZM. Hinzu kommen dann noch Informationen über Auslandsaufenthalte und Hilfsorganisationen sowie eine kritische Bestandsaufnahme des BZÄK-Vizepräsidenten, Prof. Dietmar Oesterreich, der sich mit dem Thema „Der Zahnarzt in der postfaktischen Zeit" auseinandersetzt.

Für Dr. Carla Kozmacs (Uni Witten/Herdecke) steht fest, dass der geeigneten Kommunikation speziell mit den Patienten im Studium zu wenig Zeit eingeräumt wird: „Das Studium bereitet perfekt auf das Legen von Füllungen, das Präparieren von Kronen oder auch auf das Scaling vor. Das alles kann vielleicht die Kursaufsicht bewerten, nicht aber der Patient. Dieser beurteilt die Kompetenz, das Einfühlungsvermögen und letztendlich auch den Erfolg der Behandlung an der Art, wie der Zahnarzt auf sie oder ihn ,wirkt'. Nach dem Studium ist die Art ,zu wirken ' die Visitenkarte des Zahnarztes." Es gebe wissenschaftliche Belege dafür, dass sich der Erwerb kommunikativer Kompetenzen im (Zahn-)Medizinstudium positiv auf das Therapieergebnis auswirkt. „Patientenzentrierte Kommunikation“ und „Motivational Interviewing" seien Hilfsmittel bei der Vermittlung kommunikativer Kompetenzen und etablierten sich gerade an ein paar wenigen deutschsprachigen Universitäten. Dr. Kozmacs erläutert weiter: „Bei der patientenzentrierten Kommunikation und dem Motivational Interviewing handelt es sich allerdings lediglich um Basistechniken der Kommunikation. Sie sind die gängigen Zutaten, um ein gewöhnliches Gulasch zuzubereiten - jeder

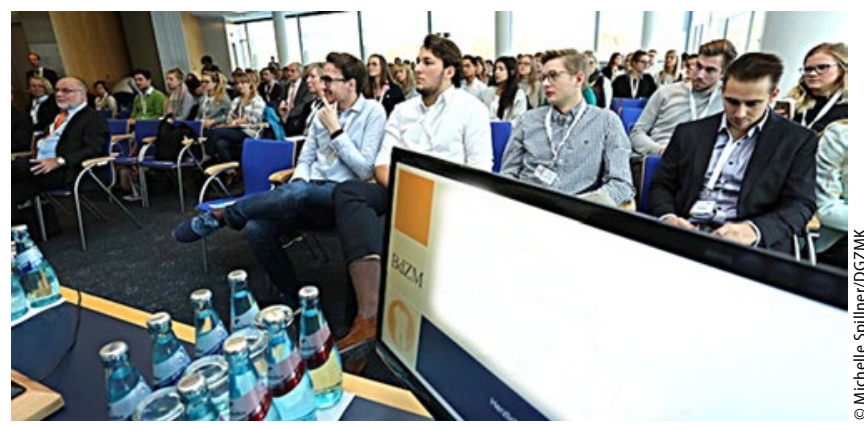

kann es erlernen. Das Besondere ist der Tiger; das Schwierige ist, ihn zu fangen. Beim Tigergulasch geht es nicht einfach um Kommunikationstechniken. Tigergulasch klingt schon ziemlich verrückt und verlangt eine andere Art zu Denken. Es macht den Umgang mit diesen Techniken bzw. den Patienten nachhaltig erfolgreich und das über die Grenzen des Behandlungszimmers hinaus. Tigergulasch ist die Grundlage nachhaltig gelungener, zwischenmenschlicher Kommunikation." Davon können sich die Teilnehmer am Studententag dann überzeugen.

Eröffnet wird der Studententag von DGZMK-Präsident Prof. Michael Walter (TH Dresden), den Abschluss bildet der Vortrag des langjährigen Berliner Kammerpräsidenten Dr. Wolfgang Schmiedel: „Pack's an, mach mit! - Zahnärztliche Hilfsprojekte in Deutschland - wie und wo kann ich helfen?"

Studierende können für die Fahrt nach Frankfurt Reisekostenzuschüsse bei der DGZMK beantragen und haben die Möglichkeit, für 15 Euro Registriergebühr den Studententag und das Programm des Deutschen Zahnärztetages zu besuchen. Infos dazu finden sich auf der Website www.dtzt.de.

Weitere Informationen, auch zur für Studierende kostenfreien Mitgliedschaft in der DGZMK, finden sich auf der Homepage www.dgzmk.de.

\begin{tabular}{|c|c|}
\hline \multicolumn{2}{|c|}{$\begin{array}{l}\text { Studententag (Spektrum 1+2) } \\
\text { Deutsche Gesellschaft für Zahn-, Mund- und Kieferheilkunde (DGZMK), } \\
\text { young dentists (yd²), Bundesverband der Zahnmedizinstudenten in } \\
\text { Deutschland e.V (BdZM) }\end{array}$} \\
\hline 10:00-10:30 & $\begin{array}{l}\text { Prof. Dr. Michael Walter, Lotta Westphal, Maximilian Voß } \\
\text { Begrüßung }\end{array}$ \\
\hline 10:30-11:15 & $\begin{array}{l}\text { Dr. Carla Kozmacs } \\
\text { Zahnärztliche Kommunikation - wie man ein Tiger- } \\
\text { gulasch kocht... }\end{array}$ \\
\hline $11: 15-12: 00$ & Pause \\
\hline $12: 00-12: 45$ & Zahnärztliche Kommunikation Teil 2 \\
\hline 12:45-14:15 & Pause \\
\hline 14:15-14:45 & $\begin{array}{l}\text { Prof. Dr. Dietmar Oesterreich } \\
\text { Der Zahnarzt in der postfaktischen Zeit }\end{array}$ \\
\hline 14:45-15:15 & $\begin{array}{l}\text { Tobias Bauer } \\
\text { Basics and more für einen Arbeitsaufenthalt im Aus- } \\
\text { land }\end{array}$ \\
\hline 15:15-15:45 & Pause \\
\hline $15: 45-16: 15$ & Famulaturberichte - Aktuelles aus 2017 (BdZM) \\
\hline $16: 15-16: 45$ & $\begin{array}{l}\text { Dr. Wolfgang Schmiedel } \\
\text { „Pack's an, mach mit!“ Zahnärztliche Hilfsprojekte in } \\
\text { Deutschland - wie und wo kann ich helfen? }\end{array}$ \\
\hline
\end{tabular}

